\title{
Metacontrast suppression and criterion content: A discriminant function analysis
}

\author{
IRA H. BERNSTEIN, SEBASTIANO A. FISICARO, and JEFFREY A. FOX \\ University of Texas at Arlington, Arlington, Texas 76019
}

\begin{abstract}
Three test and three mask energies of a metacontrast display were varied orthogonally and randomly over trials. The stimulus onset asynchrony (SOA) separating them was varied over blocks of trials from 0 to $180 \mathrm{msec}$ in $30-\mathrm{msec}$ steps. Both the accuracy in judging the test and the coherence (consistency) of the judgments were U-shaped functions of SOA. Thus, metacontrast suppression is in part due to inadequate information. In addition, mask energy was found to correlate negatively with judgments of the test at short SOAs but positively at longer SOAs. This indicates that part of the masking effect is due to inappropriate use of information. Certain similarities were noted between these findings and those obtained with judgments of frequency in the auditory-recognition masking paradigm. In general, the results indicate that subjects respond to different features of the stimulus situation as SOA varies.
\end{abstract}

Some years ago, Hake and Rodwan (Hake, Faust, McIntyre, \& Murray, 1967; Hake \& Rodwan, 1966; Hake, Rodwan, \& Weintraub, 1966; Rodwan \& Hake, 1964) applied the linear discriminant function (LDF) to the study of perception. The LDF is mathematically defined as that weighting of a set of independent variables which maximally differentiates groups in the sense of maximizing the variance between groups relative to the variance within groups. In Hake and Rodwan's application, a psychophysical observation is considered a point in $\mathrm{N}$ space defined by the dimensions of stimulation. The response categories are a set of centroids (ideal points) in this space. Classification of a given observation proceeds on the basis of the distances between the observation and each of the centroids, i.e., the subject chooses that response category which is in some sense "closest" to the observation. Thus, in the general application of the LDF, dimensions of stimulation correspond to the independent variables and the response categories correspond to groups.

Quite often, two further constraints exist in the type of experiment considered by Hake and Rodwan: (a) the subject is requested to respond along a single dimension, and (b) one, and only one, of the dimensions of stimulus variation is designated as relevant (to be responded to) and the remaining dimensions are designated as irrelevant (to be ignored). For example, Hake et al. (1967) had subjects judge the size of a small square placed within a larger square. Inner and outer square sizes varied orthogonally at

Supported in part by a grant from the Organized Research Fund of the University of Texas at Arlington and the Genco Abbandando Foundation. We are indebted to three reviewers and Professor Bruno Breitmeyer for their most cogent comments. A portion of these data was presented at the 1976 Convention of the Association for Research in Vision and Ophthalmology, Sarasota, Florida. Requests for reprints should be addressed to Ira H. Bernstein, Department of Psychology, University of Texas at Arlington, Arlington, Texas 76019. three levels each in various experimental conditions. The conditions differed in the range of outer square variation relative to inner square variation. The outer square was of constant size and absent in two control conditions, respectively. In all cases, the task was to classify the size of the inner square as "small," "medium," or "large."

Experiments of the general form of Hake et al. (1967) had been conducted in many previous instances. In these prior experiments, though, the only dependent variable studied was accuracy, typically defined as the percentage of correct responses. Hence, it was not possible to distinguish between two sources of judgmental error. In the one, the subject's usage of information is inappropriate, whereas in the other, the subject's information is inadequate. In the extreme case of chance performance, these errors could arise because the subject pays attention to the outer and not the inner square or because the subject fails to pay attention to the display, respectively. These two sources of error are not mutually exclusive; hence, either or both forms of error may arise.

Application of the LDF to the data allowed this distinction between inadequate and inappropriate information to be made operationally. Basically, inappropriate responding occurs when the LDF projects onto an irrelevant dimension. The extent of inappropriate responding can be defined in terms of the slope (or angle) between the LDF and the relevant axis. Likewise, one can compute the coherence, or systematicity of responding, in terms of the separation among response categories along the LDF independently of accuracy, or separation among categories as projected onto the relevant dimension. Inadequate responding occurs when there is a decrease in the coherence from some appropriately chosen baseline.

In the Hake et al. (1967) experiment, introduction of the outer square as an irrelevant contextual variable 
did induce inappropriate judgments, because all subjects showed at least some tendency to respond to it as well as to the inner square. However, while accuracy decreased for all three subjects as outer square variation increased, the coherence decreased for only one of the subjects. The remaining subjects maintained constant coherence by responding progressively more to the outer square, i.e., by responding inappropriately.

Another aspect of the LDF is that different distance models can be used to infer how the subject combines relevant and irrelevant information when inappropriate responding occurs. One possibility is that the subjects respond only to the single dimension defined by the LDF which, in the Hake et al. (1967) experiment, would be a weighted difference between inner and outer squares. In this case, the weighted difference alone is sufficient to determine the response. This was termed a nondiscriminating observer model. In contrast, a subject might employ the context furnished by irrelevant information in an attempt to eliminate some of the randomness in perception, but the attempt might not be totally successful. This would correspond to making separate judgments of the inner and outer squares and using the latter as a covariate. The authors termed this a discriminating observer model.

The distinction between these two models may be restated in information processing terms. The nondiscriminating model states that raw stimulus input is judged along a single dimension without attempting to separate out relevant and irrelevant information. The experimentally defined dimensions are not, in fact, treated as such psychologically, and the subject's resulting perception is univariate. Hence; sensory processes (stimulus encoding) and cognitive processes (analysis of the resultant along a decisional axis) occur at the same functional level. Conversely, the discriminating observer model states that information is encoded separately for each dimension and then combined at a second, higher, level to form the decision axis. The experimentally defined dimensions are also psychological dimensions, and the subject's perception is truly multivariate. This distinction is similar in many ways to Garner's (1974) use of the terms integral and separable, respectively, although the empirical techniques Hake et al. (1967) used were quite different, being based upon various terms derived from the LDF analysis.

Hake et al. (1967) found that the discriminating observer model fit one of their three subjects and the nondiscriminating observer model fit the remaining two subjects. The accuracy of all subjects was quite similar in the sense that it was best in the constant outer square control condition and poorest in the most variable experimental condition. Since performance was intermediate in the case where the outer square was absent and consequently failed to provide context, it may be said that context can either help or hinder accuracy. Furthermore, the manner in which context was used in the nondiscriminatingdiscriminating sense was under the subject's control.

At the same time that Hake and Rodwan's work appeared, Kahneman (1968) reviewed the metacontrast literature. He emphasized the need to infer the subject's criterion content, defined as a "description of the code that a subject uses in mapping his private experience onto responses to the experimenter's question" (p. 410). This suggestion was apparently made independently of Hake and Rodwan's work.

The LDF and the concept of criterion content, as originally used by Kahneman, are polar opposites in several ways. The former is quantitative, does not require extensive verbal description of the subject's sensations, relies upon particular experimental manipulations (typically, orthogonal variation of relevant and irrelevant dimensions), and is normally (but not of necessity) applied to accuracy judgments. The latter is qualitative, requires extensive verbal descriptions, does not rely upon any specific experimental manipulation beyond those normally used to establish the phenomenon, and is normally (but not of necessity) applied to phenomenal judgments. Yet, in a far more significant sense, the two do in fact relate to the same issue, namely that a subject may respond to different aspects of the stimulus environment as a given variable is manipulated. Hence, in the more general, and perhaps more heuristic sense, the LDF might better be viewed as a partial definition of criterion content. This is because the LDF describes the relative contribution of each of several sources of stimulation to the judgment, surely one way to conceptualize a code in Kahneman's sense. ${ }^{1}$

The LDF would seem particularly useful to study the role of criterion content in metacontrast. Despite wide differences in approach, students of this phenomenon have payed little heed to Kahneman's request. Perhaps this is due to the difficulties encountered in making use of extensive verbal descriptions. There are also other difficulties with his original suggestion that are not found with the LDF, e.g., one cannot assume that subjects use the same verbal labels to describe common sensory experiences, and vice versa. The reason that something more than a univariate description of performance is needed is that brightness is not the only sensation to change with stimulus onset asynchrony (SOA) separating test from mask. As Kahneman $(1967,1968)$ and others (e.g., Fehrer \& Smith, 1962) have noted, the mask in a metacontrast experiment produces conflicting apparent-motion cues. Perhaps these motion cues are insufficient to explain metacontrast suppression (Weisstein \& Growney, 1969), but they illustrate the complex sensory effects of introducing a mask and should not be ignored in analyzing the phenomenon.

In this paper, the use of the LDF to study the cri- 
terion content of metacontrast is described. Structurally, the experiment is isomorphic to Hake et al. (1967) in that the experimental conditions involved orthogonal variation at three imperfectly discriminable levels along the relevant and irrelevant dimensions. The relevant dimension was the energy of the test and the irrelevant dimension was the energy of the mask. The SOA defined the experimental conditions. In the control condition, stimulation was univariate in the sense that no mask was present. No fixed-energy mask control condition was employed. This is because Bernstein, Proctor, Belcher, and Schurman (1974) have already found that accuracy is higher with a concurrent, fixed-energy mask than with no mask.

In all cases, the subjects were instructed to classify the test as "bright," "medium," or "dim" to maximize accuracy and had some experience with the metacontrast literature through courses in perception. However, additional subjects were run who were theoretically naive and trained to report the test phenomenally. Their data are indistinguishable from those of the subjects to be reported.

\section{METHOD}

\section{Subjects}

Four graduate student volunteers enrolled at the University of Texas at Arlington served as subjects. All had either normal or corrected-to-normal eyesight.

\section{Apparatus and Stimuli}

Stimuli were presented by transillumination on a four-channel Iconix tachistoscope (Model 6192) using Sylvania bulbs (F8TS/D). Viewing was monocular through a 2-mm artificial pupil. A Spectra spotmeter (Model UBD-1) was used to verify luminance levels, and a Tektronix oscilloscope (Model 564) was used to verify timing accuracy. A telegraph key was used in order for subjects to selfinitiate trials after both experimenters indicated that the necessary adjustments had been made. The two experimenters and the subject sat in the same room.

A small circle that remained on continually was used for fixation. It was contained in Exposure Field IV, but illuminated by a separate tungsten source which did not affect the other contents of the field. The fixation circle was sufficiently dim so as to be barely visible to a light-adapted subject. It became clear with dark adaptation and, for this reason, served as a control over adaptation effects.

Exposure Field I was used for the three test stimuli (TS)-dim TS, medium TS, and bright TS. Each square appeared $.51 \mathrm{deg}$ of visual angle above the fixation circle. Exposure Fields II, III, and IV housed the three masking stimuli (MS) each of which consisted of a pair of squares whose sides were adjacent to the TS. The length of each side of TS and MS was $1.0 \mathrm{deg}$ of visual angle. The location of each MS with respect to the three fields was changed during the experiment so that each MS appeared equally often in each exposure field. This was done to eliminate coloration and other cues specific to each field from providing information.

The TS and MS durations were both $50 \mathrm{msec}$. The three TS were changed manually by one of the two experimenters by dropping Kodak neutral density filters into Exposure Field I. The other experimenter both selected the MS appropriate for that trial by means of a toggle switch, which enabled one of the three exposure fields (II, III, or IV) to fire, and recorded the subject's responses on an answer sheet. Luminances for bright TS and MS, medium
TS and MS, and dim TS and MS were 64,40 , and $25 \mathrm{~cd} / \mathrm{m}^{2}$, respectively (i.e., $0.0,-.2$, and $-.4 \log$ units referenced to the bright TS and bright MS).

\section{Procedure}

The subjects first pariticpated in several practice sessions, during which they judged the brightness of the three TS energies alone (i.e., no mask) until their accuracy had apparently reached asymptote. The accuracies in terms of percent correct responses at the end of practice ranged from $67 \%$ to $75 \%$ and were within binomial error of one another for two consecutive sessions. At the beginning of each experimental session, the subjects were darkadapted for $10 \mathrm{~min}$, after which they received 15 warm-up trials on TS alone with feedback. These data were not used further. Furthermore, just prior to the presentation of each SOA, the subjects were given a few familiarization trials at that SOA.

During the experiment, 270 trials were run at each of seven SOAs $(0,30,60,90,120,150$, and $180 \mathrm{msec})$ comprising the experimental conditions and 90 trials were run in a no-mask control condition. The 270 experimental trials per SOA were divided into three 90 -trial blocks. Within each experimental block, each of the nine stimulus alternatives ( 3 TS energies $\times 3$ MS energies) appeared 10 times. Likewise, the 90 control trials were divided into three 30-trial blocks. Within each control block, each of the three TS energies appeared 10 times. One experimental block per SOA and a control block were run in random order to form a cycle of eight blocks. This process was repeated to form two more cycles. Different randomizations were used within each cycle and across subjects. Each subject was run in eight 1 -h sessions with an average of three blocks per session.

The experimenters began each trial with appropriate dial settings and switch adjustments, including dummy dial settings and filter drops to avoid giving information to the subject. One experimenter then signaled the subject to initiate the trial, whereupon the subject responded "bright," "medium," or "dim" with respect to the apparent intensity of the TS. A rest break was given after each block.

\section{RESULTS}

The first analysis of the data was to assign the values " 1 ," " 2 ," and " 3 " to the "bright," "medium," and "dim" response categories, respectively, and to compute the average response at each SOA. In an accuracy task with equiprobably high, medium, and low test energies, standard decision theoretic considerations suggest that responses should be divided equally among the three categories. Hence, the average response should be close to 2.0 at each SOA. This was, in fact, the case. The grand mean over subjects and SOAs was 1.93. An analysis of variance (7 SOAs + no mask $\times 4$ subjects) indicated that the means did not differ from one another, $F(7,21)=$ $1.98, \mathrm{p}>.05$.

The LDFs were determined in the manner described by Hake et al. (1967) for each of 28 cases (7 SOAs $x$ 4 subjects) using log energy as the metric. The quantities employed were the bivariate response centroids for the three response categories, the within-responseclass sum-of-products matrix (W), and the betweenresponse-class sum-of-products matrix (B). Two LDFs may be obtained from each cell, corresponding to the two eigenvectors of $\mathrm{W}^{-1} \cdot \mathrm{B}$. The first LDF 


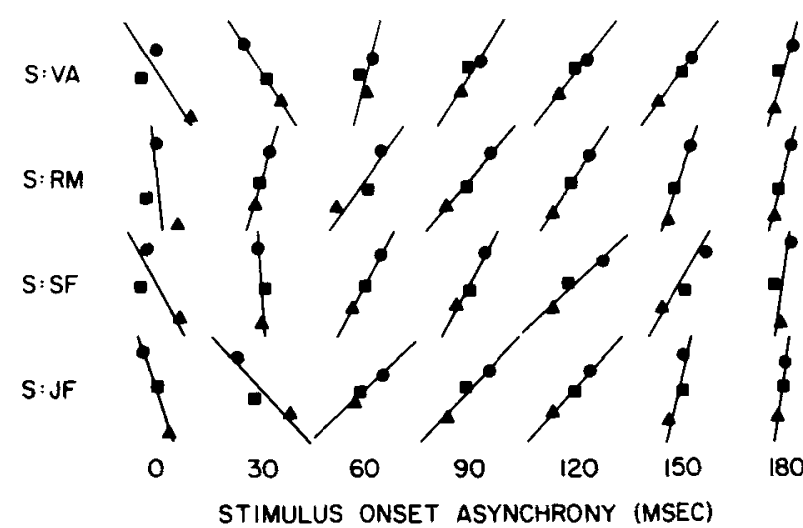

Figure 1. Individual discriminant axes and centroids for each stimulus onset asynchrony (SOA). (Note-The range of each axis is from 0 to -.4 in both directions; circles = "bright" centroids, squares $=$ "medium" centroids, and triangles = "dim" centroids.)

accounts for at least $99 \%$ of the trace in 21 of the 28 cases. This indicates that the centroids are colinear in the space, as required by the instructions to treat the response categories as a continuum. The centroids and associated first LDFs are presented in Figure 1.

The analysis provides univariate $F$ ratios for the two dimensions taken separately to see whether the LDF differs significantly from being vertical (responses made solely to the relevant dimension) or horizontal (responses made solely to the irrelevant dimension). The contribution of the relevant dimension was significant in all 28 cases beyond the .01 level. The contribution of the irrelevant dimension was significant beyond the .01 level in 20 cases and beyond the .05 level in an additional 5 cases, all of which were at $150-$ or $180-$ msec SOA. The irrelevant dimension failed to contribute significantly in two cases at 30-msec SOA (subjects R.M. and S.F.) and in one case at 60-msec SOA (subject V.A.). Hence, in general, it can be stated that subjects' judgments of the test were influenced by both test and mask energy, i.e., their criterion content was some combination of both energies, and the respective weightings varied over SOA. Thus, metacontrast is accompanied by inappropriate responding.

However, as Figure 1 also indicates, the manner in which information about the mask was used was quite different at short (0- and $30-\mathrm{msec})$ vs. long $(60-\mathrm{msec}$ or more) SOAs. In the former case, the weighting of MS energy in the response is negative in the sense that high mask energies lead to "dim" responses (as can be seen from the negative slopes of the LDFs). In the latter case, precisely the reverse is true, since the slopes are positive. The negative slopes are consistent with the effects of simultaneous contrast, which, in turn, is attributable in some theoretical frameworks to lateral inhibition. Clearly, the positive slopes are not explicable in terms of either simultaneous contrast or lateral inhibition. Rather, it suggests that subjects are responding to the total (or equivalently, the average) energy while poorly differentiating the appropriateness of the source.

The coherence of the judgments at a given SOA may be defined as the ratio of the between-groups sum of squares to the within-groups sum of squares measured along the discriminant. It is obtained from the quadratic form of $\mathrm{W}^{-1} \cdot \mathrm{B}$ weighted by the LDF. Likewise, the accuracy of the judgments at a given SOA may be defined as the corresponding ratio measured along the relevant axis and is computed from the quadratic form of $\mathrm{W}^{-1} \cdot \mathrm{B}$ using the weighting vector $(1,0)$. Figure 2 contains the mean coherence over subjects and the mean accuracy over subjects as a function of SOA. Also presented is the mean accuracy inferred from the no-mask control over subjects. As can be seen, both coherence and accuracy functions are clearly U-shaped, and the accuracy function begins slightly above the no-mask control. Of necessity, the coherence will be at least as great as the accuracy.

An analysis of variance of the coherence measures ( 7 SOAs $\times 4$ subjects) indicated that the main effect of SOA was significant, $F(6,18)=4.23, p<.05$. The linear and quadratic components accounted for $21 \%$ and $71 \%$ of the trend, $F s(1,18)=5.30$ and 18.13 , ps $<.05$ and .01 , respectively. Analysis of variance of the accuracy measures ( 7 SOAs + no mask $\times 4$ subjects) indicated that the main effect was significant, $F(7,21)=5.38, p<.01$. The linear and quadratic components and the mask/no-mask difference accounted for $9 \%, 60 \%$, and $21 \%$ of the effect, $\mathrm{Fs}(1,21)=3.30,33.42$, and 10.69 , n.s. and $\mathrm{ps}<.01$, respectively.

Figure 3 contains the goodness-of-fit data in terms of the mean percent variance explained by each of the two distance models, nondiscriminating and discriminating. As can be seen, the discriminating model consistently fits the data somewhat better. However, this model also employs more free parameters. The tradeoff implied between goodness-of-fit and statis-

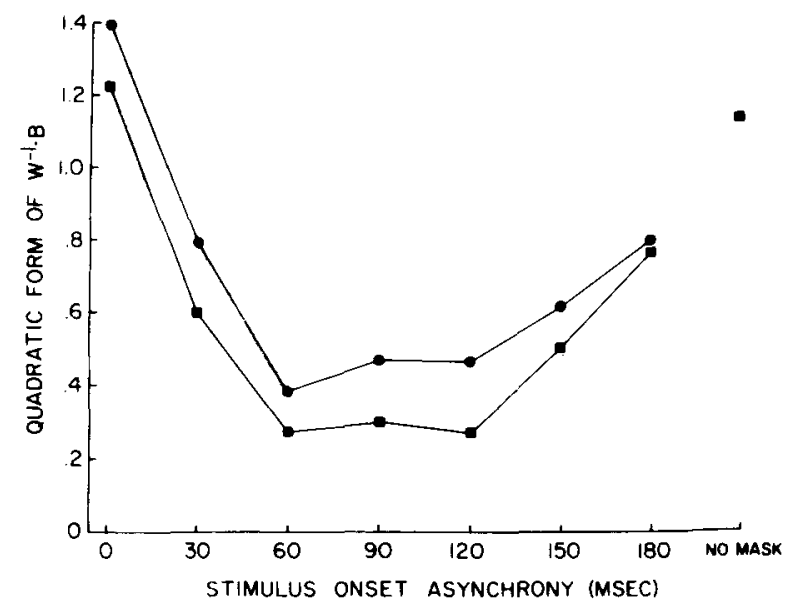

Figure 2. Coherence (circles) and accuracy (squares) as a function of stimulus onset asynchrony (SOA). 


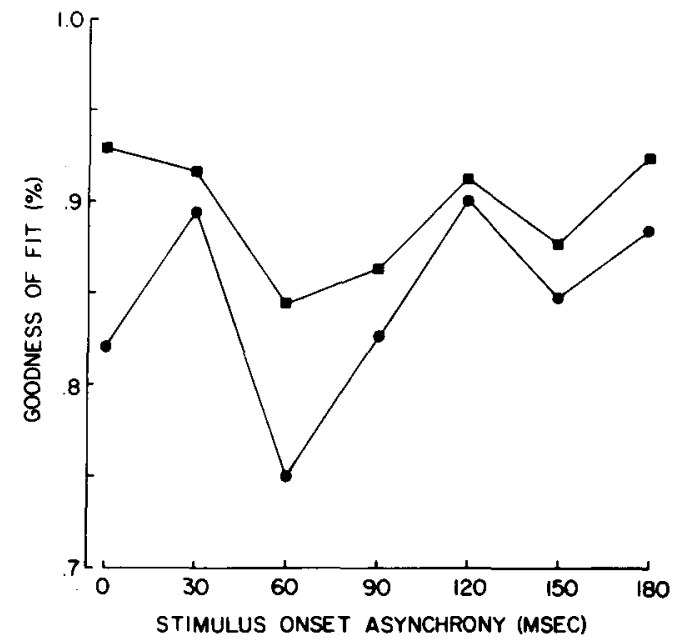

Figure 3. Goodness-of-fit for the discriminating model (squares) and the nondiscriminating model (circles) as a function of stimulus onset asynchrony (SOA).

tical parsimony is, of course, not peculiar to the present situation.

The drop in the nondiscriminating function at $60-\mathrm{msec}$ SOA is descriptive of only two of the four subjects. Save for this point, the difference between curves is relatively constant. The goodness-of-fit to the discriminating observer model was similar to the fit reported by Hake et al. (1967), but the fit to the nondiscriminating observer model was generally better than they reported. Analysis of variance of the percentage of variance explained (nondiscriminating vs. discriminating model $\times 7$ SOAs $\times 4$ subjects) indicated that the difference between models was the only significant source of variation, $\mathrm{F}(1,18)=18.14$, $\mathrm{p}<.01$. Unfortunately, the additional variance accounted for by the discriminating model is not additive with respect to the variance accounted for by the nondiscriminating model so that the specific effects of the additional parameters cannot be tested. The nondiscriminating model can fit the data better than the discriminating model, and did, for some subjects at longer SOAs.

To summarize these results: (a) subjects reacted to the difference between test and mask energy at short SOAs but to the sum at longer SOAs; (b) regardless of whether the adequacy of perception was defined in terms of accuracy or coherence, U-shaped masking was obtained ${ }^{2}$; and (c) the discriminating observer model provided a slightly better fit to the data than the nondiscriminating observer model; but (d) the latter provided an excellent representation of the subject's judgmental behavior and employs fewer free parameters.

\section{DISCUSSION}

The transition from reactions based upon a difference between test and mask energy to reactions based upon the sum as SOA increases has been obtained previously with different methodology (Bernstein et al., 1974). Hence, it is not an artifact of the LDF methodology. One main difference between the earlier study and the present one is that the former used two rather than three test and mask energy levels. Nonetheless, Figure 3 of that paper illustrates that the dim mask led to more "bright" responses than the bright mask at short SOAs but led to more "dim" responses at long SOAs.

This transition in criterion content is probably not limited to brightness masking, since it also seems to exist in auditory masking of frequency information. Several years ago, Egan and Meyer (1950) found that the pitch of a sinusoidal test tone was displaced away from the center frequency of a concurrent narrow-band noise mask. Had they presented the mask at frequencies above and below that of the test, displacement would have led to the type of negative correlation presently observed.

More recently, Hawkins and Presson (in press) did, in fact, find a negative correlation using sinusoidal tests and masks. However, they found that this held only up to 20 - or $30-\mathrm{msec}$ SOA and when test and mask reached the same ear, i.e., under monaural or binaural, but not dichotic, presentation. The failure to find a negative correlation using dichotic stimulation indicates that this displacement effect is peripheral.

On the other hand, Hawkins and Presson (in press) found a positive correlation between judgments of the test and mask frequency at longer SOAs. This held regardless of the relation between the ear stimulated by the test and the ear stimulated by the mask as long as this relation was unpredictable, i.e., as long as subjects could not use location foreknowledge to employ peripheral gating. Hence, this positive correlation had to be more central than the effect originally reported by Egan and Meyer (1950).

Since we used only monocular stimulation, we cannot see if the same inferences may be drawn in vision at this point in our studies. If they can, it would suggest that the transition from negative to positive correlation also involves a changing contribution of peripheral and central factors as SOA changes in metacontrast.

Hawkins and Presson (in press) explicitly suggest that their effects should hold in both auditory and visual masking. The present data are in support of this supposition. The negative correlation can be accounted for by the same mechanisms generally proposed for simultaneous contrast.

We previously ncted that inappropriate information utilization can be inferred from an oblique discriminant. However, it must be added that a subject can employ information inappropriately yet achieve a lower error rate than would be obtained by using an alternative strategy that did not lead to inappro- 
priate responding. As stated previously (Bernstein, Proctor, Proctor, \& Schurman, 1973; Bernstein et al., 1974; Bernstein, Smith, \& Adey, in press), we suggest that the strategy used at short SOA was to compare test and mask rather than judge the test independently of the mask, as would be necessitated at long SOA or in the "no-mask" control. The term "simultaneous contrast" implies a comparison at some level. Using information in this way may cause subjects to misperceive the test because they misperceive the mask. Even so, accuracies with concurrent testmask presentation were generally higher than they were at long SOA or in the "no-mask" case. This is consistent with the more general point that comparative judgments are more accurate than absolute judgments when the signal/noise ratio is high. This issue has been discussed in detail by Hake et al. (1966, 1967).

The explanation that Hawkins and Presson (in press) gave to their positive correlation was that the subjects simply could not separate information about the test from information about the mask. Because vision is more spatially organized than audition, confusion would seem less likely in metacontrast. The magnitude of these effects may well be less here since we cannot compare the two studies directly. In addition, though, it is reasonable to assume that the judgment of the test is not an instantaneous process. During the time required to gather the necessary information, the mask would be presented and potentially interact with the decaying trace of the test. Interocular light scatter is a specific possible source of confusion as to the locus of stimulus change. Of perhaps greater importance is the possible confusion caused by apparent movement between test and mask. Apparent movement clearly makes it difficult to isolate where a particular stimulus change occurred.

The finding that the discriminating observer model fit the subjects' response distributions better than the nondiscriminating observer model suggests that information about the test and information about the mask were extracted separately at one level and combined at a higher level to be classified along a decision axis. Had the nondiscriminating model fit as well as the discriminating model, one stage would suffice for explanation. It would not be known, however, whether this single stage was sensory (Breitmeyer \& Ganz, 1976; Weisstein, 1968, 1972) or cognitive (Uttal, 1970, 1971). As previously indicated (Bernstein et al., 1973, 1974), metacontrast suppression seems to involve both sensory and judgmental/cognitive factors and not either one alone. It needs to be reiterated, though, that a single stage representation is not bad in an absolute sense.

It is useful to consider how a neurophysiological model like Breitmeyer and Ganz's (1976) would account for these results. Their model treats metacontrast suppression as the result of inhibition of the test's sustained activity by the mask's transient activity. Because transient activity, in general, has a shorter latency than sustained activity, the magnitude of this inhibition is greatest when the mask is delayed. These assumptions are insufficient to account for the positive correlation found at long SOAs.

Breitmeyer (Note 1) suggested the following, based upon a study by Breitmeyer and Ganz (Note 2). The latter presented evidence that input channels are characterized by a damped oscillating temporal response, i.e., an alternating series of positive and negative phases. The mask's transient activity could enhance the negative phase of the test's sustained activity. If the subjects judged the difference between the initial positive and secondary negative peaks, a successive contrast-like effect could lead to the positive correlation. However, even if one accepts this or related interpretations (Weisstein, 1968, 1972), one needs ask what parameters of the neural response determine the judgment, i.e., to determine the subject's criterion content. All theories of this general form predict more than a simple attenuation of the neural response, because they predict qualitative changes in the shape of the neural response as SOA varies. In short, even these theories imply that a subject may be responding to different features of input as SOA varies.

\section{REFERENCE NOTES}

1. Breitmeyer, B. Personal communication, May 1976.

2. Breitmeyer, B., \& Ganz, L. Temporal integration in spatial frequency channels. Paper presented at the meeting of the Association for Research in Vision and Opthalmology, Sarasota, Florida, Spring, 1976.

\section{REFERENCES}

Bernstein, I. H., Proctor, R. W., Belcher, J., \& Schurman, D. L. An analysis of U-shaped metacontrast. Perception \& Psychophysics, 1974, 16, 329-336.

Bernstein, 1. H., Proctor, J. D., Proctor, R. W., \& Schurman, D. L. Metacontrast and brightness discrimination. Perception \& Psychophysics, 1973, 14, 293-297.

Bernstein, I. H., Smirh, D. B., \& Adey, M. Perceptual and response interdependencies in visual masking. In $S$. Dornic \& A. F. Sanders (Eds.), Attention and performance, VI. Potomac, Md: Erlbaum Associates, in press.

BreitmeYer, B., \& GANz, L. Implications of sustained and transient channels for theories of visual pattern masking, saccadic suppression, and information processing. Psychological Review, $1976,83,1-36$.

Egan, J. P., \& Meyer, D. R. Changes in pitch of tones of low frequency as a function of the pattern of excitation produced by a band of noise. Joumal of the Acoustical Society of America. $1950,22,827-833$.

FEHRER, E., \& SMITH, E. Effect of luminance ratio on masking. Journal of Experimental Psychology, 1962, 63, 143-147.

GARNER. W. R. The processing of information and structure. Potomac, Md: Erlbaum Associates, 1974.

GARNer, W. R., \& Morton, J. Perceptual independence: Definitions. models, and experimental paradigms. Psychological Bulletin. 1969. 73, 233-259. 
Hake, H. W., FAust, G. W., McIntyre, J. S., \& Murray, H. G. Relational perception and modes of perceiver operation. Perception \& Psychophysics, 1967, 2, 469-478.

Hake, H. W., \& Rodwan, A. S. Perception and recognition. In J. B. Sidowski (Ed.), Experimental methods and instrumentation in psychology. New York: McGraw-Hill, 1966.

Hake, H. W., Rodwan, A. S., \& Weintraub, D. J. Noise reduction in perception. In $K$. R. Hammond (Ed.), The psychology of Egon Brunswik. New York: Holt, Rinehart, \& Winston, 1966.

Hawkins, H. L., \& Presson, J. C. Masking and preperceptual selectivity in auditory recognition. In S. Dornic \& A. F. Sanders (Eds.), Attention and performance VI. Potomac, Md: Erlbaum Associates, in press.

Kahneman, D. An onset-onset law for one case of apparent motion and metacontrast. Perception \& Psychophysics, 1967, 2, $557-583$.

KafNEmAN, D. Method, findings, and theory in studies of visual masking. Psychological Bulletin, 1968, 70, 404-425.

Matin, E. The two transient (masking) paradigm. Psychological Review, 1975, 82, 451-461.

Rodwan, A. S., \& Hake, H. W. The discriminant function as a model for perception. American Journal of Psychology, 1964, 77, 380-392.

UTTAL, W. R. On the physiological basis of masking with dotted visual noise. Perception \& Psychophysics, 1970, 7, 321-327.

UTTAL, W. R. The psychobiological silly season-or-what happens when neurophysiological data become psychological theories. Journal of General Psychology, 1971, 84, 151-166.

Weisstein, N. A. A Rashevsky-Landahl neural net: Simulation of metacontrast. Psychological Review, 1968, 75, 494-521.

Weisstein, N. A. Metacontrast. In D. Jameson \& L. M. Hurvich (Eds.) Handbook of sensory physiology (Vol. 7, Part 4) Visual psychophysics. Berlin: Springer-Verlag, 1972.

Weisstein, N., \& GRow NEY, R. L. Apparent motion and metacontrast: A note on Kahneman's formulation. Perception \& Psychophysics, 1969, 5, 321-328.

\section{NOTES}

1. A reviewer has noted that there is a distinction between multiple sources of stimulus information whose weightings may be inferred via the LDF and the multiple concurrent sensory effects of one or more stimulus inputs. The latter is also certainly appropriate to the assessment of criterion content. We have previously used multiple uncertainty analysis (Garner, 1974; Garner \& Morton, 1969) to study these multiple concurrent effects in nonmetacontrast masking (Bernstein, Smith, \& Adey, in press) and are currently using it in a metacontrast experiment. Consequently, we conceive of the LDF as capturing the meaning of the term "criterion content" in an important, but limited, way.

2. The present response measures and those normally reported in metacontrast studies need to be distinguished. The present measures do not assess how bright the test appeared at different SOAs. Rather, they describe the rules by which subjects classified stimuli. Indeed, the typical "bright" response to a test at 60-msec SOA may well have been evoked by a dimmer sensory experience than the typical "dim" response at shorter or longer SOAs. The subjects' knowledge of the stimulus presentation set and the desire to satisfy the accuracy criterion led them to use the three response categories with nearly equal frequency at each SOA. However, as previously noted, instructing a separate group of subjects to report the stimuli as they appeared, i.e., to make phenomenal judgments, did not lead to any differences in the form of the masking function. For this reason, as well as that of parsimony, it seems reasonable to conclude that the same factors lead to suppression of our measures and of traditionally used measures.

3. Matin (1975), apparently alone among neurophysiologically oriented investigators, has noted that test and mask may have different effects presented together than they do alone. Her "whole is different from the sum of its parts" emphasis upon the interactive nature of test and mask fits in well with our emphasis on the misleading nature of some of the cues produced by the relation of test to mask (Bernstein et al., 1973, 1974).

(Received for publication March 3, 1976; revision accepted June 25,1976 .) 